\title{
SPECTRAL ASPECTS OF THE SKEW-SHIFT OPERATOR: A NUMERICAL PERSPECTIVE
}

\author{
ERIC BOURGAIN-CHANG
}

\begin{abstract}
In this paper we perform a numerical study of the spectra, eigenstates, and Lyapunov exponents of the skew-shift counterpart to Harper's equation. This study is motivated by various conjectures on the spectral theory of these 'pseudorandom' models, which are reviewed in detail in the initial sections of the paper. The numerics carried out at different scales are within agreement with the conjectures and show a striking difference compared with the spectral features of the Almost Mathieu model. In particular our numerics establish a small upper bound on the gaps in the spectrum (conjectured to be absent).
\end{abstract}

Key Words: Schrödinger operator, skew-shift, spectrum, localization

\section{INTRODUCTION}

The almost Mathieu operator is the self-adjoint operator acting on $\ell^{2}(\mathbb{Z})$ defined by

$$
\left[H_{\lambda, \omega, \theta} u\right](n)=2 \lambda \cos (2 \pi(n \omega+\theta)) u_{n}+u_{n+1}+u_{n-1}
$$

where $\omega, \theta \in \mathbb{T}=\mathbb{R} / \mathbb{Z}$ and $\lambda$ is the coupling parameter. We always assume that $\omega$ is irrational (and later on, it is diophantine), so the potential in 1.1) is almostperiodic. A discussion of the physical motivation and background of (1.1) may be found in $\mathrm{L}$ for instance. Let us recall that $H_{\lambda, \omega, \theta}$ is a model for the Hamiltonian of an electron in a one-dimensional lattice, subject to a potential, and is also related to the Hamiltonian of an electron in a two-dimensional lattice subject to a perpendicular magnetic field. Such models go back to the work of Peierls [P] related to the theory of the quantum Hall effect where 1.1) describes a Bloch electron in a magnetic field. The case $\lambda=1$ is particularly important and called Harper's equation. From the mathematical side, 1.1 has been extensively studied over the recent decades and the complete spectral theory in the different regimes is understood (the main features will be summarized later). An important property of (1.1) is the so-called Aubry duality relating the regimes $|\lambda|<1$ and $|\lambda|>1$. A major break-through in this area came with the work of Jitomirskaya [J].

To be noted is that the theory of almost periodic Schrödinger operator has been developed in far greater generality than 1.1) (many of its specific properties are in some sense 'special') but we will not further elaborate on this here. Our interest 
goes to the formally related Hamiltonian

$$
[H u](n)=2 \lambda\left(\cos \left(2 \pi n^{2} \omega\right)\right) u_{n}+u_{n+1}+u_{n-1}
$$

which we refer to as the skew shift Schrödinger operators, since its potential can be generated from the orbits of the skew shift $T_{\omega}$ acting on $\mathbb{T}^{2}$, defined by $T_{\omega}(x, y)=$ $(x+y, y+\omega)$. Such models are relevant to the theory of the quantum-kicked rotor, the quantum version of the classical Chirikov standard map. More generally, Jacobi matrices on $\mathbb{Z}_{+}$of the form

$$
[H u](n)=2 \lambda\left(\cos \left(n^{\beta}\right)\right) u_{n}+u_{n+1}+u_{n-1}
$$

with $\beta>1$ were considered in the works of Griniasty-Fishman $\mathrm{G}-\mathrm{F}$ and BrennerFishman B-F (written in the form (1.3), assuming $\beta$ not an integer). As these authors point out, there is a large variety of potentials that are neither periodic nor incommensurate and the study of deterministric 'pseudo-random' systems is of broad interest beyond quantum chaos, including to theoretical computer science. It is believed that the spectral theory of 1.2 and 1.3 , at least for $\beta>2$, resembles that of Schrödinger operator with a random potential, even at small disorder $\lambda$. They were proposed in $\mathrm{G}-\mathrm{F}, \mathrm{B}-\mathrm{F}$. as 'pseudo-random' models and discussed heuristically. So far, the main rigorous results for $(1.2)$ assume $|\lambda|$ large. See in particular H. Krüger's paper [K1] and the related references. For $0<|\lambda| \leq 1$, there are only a few contributions and they relate either to somewhat atypical frequencies $\omega$ or modifications of 1.2 (see [K2] and the discussion in that paper). Thus at this point, there is no satisfactory mathematical theory that explains the expected phenomenology (which will be stated explicitly in $\S 3$ ).

Taking $\omega=\frac{1+\sqrt{5}}{2}$ the golden mean ratio, the purpose of this Note is to carry out some numerics related to the spectrum and eigenstates of the operator

$$
[H u](n)=2\left(\cos 2 \pi n^{2} \omega\right) u_{n}+u_{n+1}+u_{n-1}
$$

which is the skew shift counterpart of Harper's equation. The interest of such numerics is two-fold. Firstly, it gives some understanding how, on a finite scale, the eigenvalue distribution and eigenvector localization compares with the Harper case

$$
[H u](n)=2(\cos 2 \pi n \omega) u_{n}+u_{n+1}+u_{n-1}
$$

Secondly, using numerics, one can in fact prove certain spectral properties of the full operator. We illustrate this with the modest example of an upperbound on the size of possible gaps (if any) in the spectrum of 1.4. Obviously larger scale numerics would likely lead to better estimates. We also indicate how, 'in principle', appropriate numerics may permit one to start the multi-scale analysis underlying the approach for large $\lambda$, in order to prove small $\lambda$ results (for instance in the context of [K1]). 
Returning to 1.3 and more generally operators of the form

$$
[H u](n)=f\left(n^{\beta}\right) u_{n}+u_{n+1}+u_{n-1}
$$

with $f$ a nonconstant, continuous periodic function on $\mathbb{R}$, the case $\beta>0, \beta \notin \mathbb{Z}$ may be studied by different methods. It was shown for instance in [K3] that for $H$ as in 1.6 ,

$$
\text { Spec } H=[\min f-2, \max f+2] .
$$

The paper is organized as follows. In the next section, we briefly review several aspects of the theory of $1 \mathrm{D}$ lattice Schrödinger operator with various types of potentials, the almost Mathieu operator and also the conjectured behavior of models with weakly mixing potentials (for instance governed by skew-shift dynamics). In Section 3, we explain how information on certain spectral parameters (such as location and gaps in the spectrum) for the full Hamiltonian $H$ may be derived from properties of the eigenvalues and eigenvectors of 'finite models' obtained by restriction of $H$ to a finite interval. In Section 4, these considerations are further specified in the context of the skew shift potential, which is our main interest in this work. The numerical implementation appears in Section 5. We compare the Harper model (the Almost Mathieu operator at the critical coupling $\lambda=1$ ) with its skew shift counterpart, with emphasize on their spectra, eigenfunction localization and Lyapunov exponents through the energy range. Section 6 summarizes the conclusions and stresses some further research perspectives.

\section{Some BACKGROUND ON DISCRETE SChrÖDINGER OPERATORS}

We only discuss the $1 \mathrm{D}$ model (on the lattice $\mathbb{Z}$ ). Thus $\mathcal{H}=\ell^{2}(\mathbb{Z})$ is the underlying Hilbert space. We consider self-adjoint operators of the form

$$
H=V+\Delta
$$

where $V$ is a diagonal operator given by a bounded potential, i.e.

$$
V=\sum_{n \in \mathbb{Z}} V_{n}\left(e_{n} \otimes e_{n}\right)
$$

with $\left\{e_{n}\right\}$ the unit vectors of $\mathcal{H}, \Delta=$ lattice Laplacian on $\mathbb{Z}$, given by

$$
\Delta=\sum_{n \in \mathbb{Z}}\left(e_{n} \otimes e_{n+1}+e_{n+1} \otimes e_{n}\right) .
$$

The potential is often introduced by evaluation of a given bounded function

$f: \Omega \rightarrow \mathbb{R}$ on the orbits of some dynamical system $(\Omega, \mu, T)$ with $T$ an ergodic measure preserving transformation. Thus

$$
V_{n}=f\left(T^{n} x\right)
$$

with $x \in \Omega$ some base point. Recall that if we start from a general bounded sequence $\left(V_{n}\right)_{n \in \mathbb{Z}}$, the format 2.4 may always be attained by a classical construction which 
consists in taking for $\Omega$ the pointwise closure in $\mathbb{R}^{\mathbb{Z}}$ of the orbit of $\left(V_{n}\right)_{n \in \mathbb{Z}}$ under the left shift $T$

$$
(T x)_{n}=x_{n+1} \text { for } x=\left(x_{n}\right)_{n \in \mathbb{Z}}
$$

and for $\mu$ a Banach invariant mean (ergodicity is then achieved by decomposing the resulting dynamical system $(\Omega, \mu, T)$ in its ergodic components).

Returning to 1.2 , there are two models extensively studied by many authors. The first is the case of a random potential

$$
V_{\omega}=\left(V_{n}(\omega)\right)_{n \in \mathbb{Z}}
$$

with $V_{n}$ independent identically distributed random variables. This is the $1 \mathrm{D}$ Anderson model which describes for instance transport in inhomogenous media (such as a metal alloy). In this situation, $H=H_{\omega}$ and its main spectral features are the following (assuming the distribution non-constant).

(2.7) For almost all $\omega$, the spectrum $\operatorname{Spec}\left(H_{\omega}\right)$ is the set

$$
\sum=[-2,2]+\text { range } V
$$

(2.8) For almost all $\omega, H_{\omega}$ has pure point spectrum and the corresponding eigenfunctions are exponentially localized i.e. decay exponentially for $|n| \rightarrow$ $\infty$.

Property (2.8) is referred to as "Anderson localization". Let us emphasize that this discussion is $1 \mathrm{D}$, which is a well understood theory (unlike in dimension $\geq 2$ where different phenomena are expected). There are many reference works, see for instance $[\mathrm{F}-\mathrm{P}$. It is convenient to replace $V$ by $\lambda V$, where $\lambda \neq 0$ is called the disorder parameter. Thus (2.8) remains true, also for small $\lambda$.

Another extensively studied model is (1.1), i.e. the almost Mathieu operator with almost periodic potential

$$
V_{n}=2 \lambda \cos (2 \pi(n \omega+\theta)) .
$$

There is a long list of contributors and contributions to this subject, but we restrict ourselves to a summary of the final state of the theory. We always assume $\omega$ irrational, implying ergodicity of the potential and $\operatorname{Spec} H_{\lambda, \omega, \theta}$ independent of $\theta$ (as a set). Say that $\omega$ is diophantine if it satisfies for some constant $C>0$

$$
\|k \omega\|>\frac{1}{C}|k|^{-C} \text { for all } k \in \mathbb{Z} \backslash\{0\} .
$$

(2.11) For $\lambda \neq 0$, Spec $H_{\lambda, \omega}$ is a Cantor set of Lebesque measure

$$
\left|\operatorname{Spec} H_{\lambda, \omega}\right|=4|1-\lambda|
$$

assuming moreover $\omega$ diophantine.

(2.13) For $0 \leq|\lambda|<1, H_{\lambda, \omega}$ has purely absolutely continuous (ac) spectrum. 
(2.14) For $|\lambda|>1$, for almost all $\theta, H_{\lambda, \omega, \theta}$ has pure point spectrum and exhibits Anderson localization.

(2.15) For $|\lambda|=1$ and almost all $\theta, H_{\lambda, \omega, \theta}$ has purely singular continuous spectrum.

See [A-J], [J-K], J], G-J-L-S] for statements (2.11), 2.12, (2.14), (2.15) respectively; (2.13) is a consequence of Aubry duality. Some of the above results were actually proven in a stronger form, but this is not important for what follows. Note also that, by 2.12), the Harper operator has spectrum of zero Lebesgue measure which forces it automatically to be Cantor.

Let us consider next the skew shift Schrödinger operator obtained by taking in 2.4 for $T$ the skew shift acting on the 2 -torus $\mathbb{T}^{2}$, i.e.

$$
T(x, y)=(x+y, y+\omega)
$$

and $f=\lambda \cos \theta$ acting on the first coordinate. Thus

$$
H_{\lambda, \omega, x, y}=2 \lambda V+\Delta \text { with } V_{n}=\cos \left(x+n y+\frac{n(n-1)}{2} \omega\right)
$$

and we assume again $\omega$ diophantine.

It is conjectured that 2.16 displays a spectral behaviour similar to the random case (2.6), also for small $\lambda$. In particular that $\operatorname{Spec} H_{\lambda, \omega, x, y}$ has no gaps and for most $x, y, H_{\lambda, \omega, x, y}$ has pure point spectrum with Anderson localization. This last statement has been proven for $|\lambda|$ sufficiently large and $(\omega, x, y) \in \mathbb{T}^{3}$ taken in a suitable set of positive measure. From this respect, there is no difference with the almost periodic case; in particular (1.1) satisfies (2.14). On the other hand, it was shown more recently in [K1], again for $\lambda$ sufficiently large (and $\omega$ satisfying the stronger DC $\left.\|k \omega\|>\frac{c}{k^{2}}\right)$, that $\operatorname{Spec} H_{\lambda, \omega, x, y}$ contains at least an interval, hence is not a Cantor set.

Returning to 2.1), the 1D lattice Schrödinger operator can be studied using the transfer matrix formalism (which is a powerful tool specific to the 1D situation). Given an arbitrary sequence $\left(u_{n}\right)_{n \in \mathbb{Z}}$, the equation

$$
H u=E u
$$

is equivalent with

$$
\left(\begin{array}{c}
u_{n+1} \\
u_{n}
\end{array}\right)=M_{n}(E)\left(\begin{array}{l}
u_{1} \\
u_{0}
\end{array}\right)
$$

where the transfer matrix $M_{n}(E) \in S L_{2}(\mathbb{R})$ is given by

$$
M_{n}(E)=\prod_{j=n}^{1}\left(\begin{array}{cc}
V_{j}-E & -1 \\
1 & 0
\end{array}\right) .
$$

Assuming $V_{n}$ given by (2.4), define

$$
L_{n}(E)=\frac{1}{n} \int \log \left\|M_{n}(E, x)\right\| d x
$$


and the Lyapunov exponent

$$
L(E)=\lim _{n \rightarrow \infty} L_{n}(E)
$$

For ergodic $T$, one has

$$
L(E)=\lim _{n \rightarrow \infty} \frac{1}{n} \log \left\|M_{n}(E, x)\right\| \quad x \text { a.s. }
$$

The Lyapunov exponent plays a prominent role in the spectral theory of $H$. Recall in particular Kotani's theorem, stating the following:

Let $(a, b) \subset \mathbb{R}$ be an interval. Then $H$ has no ac spectrum in $(a, b)$, i.e.

$$
\sum_{a c}(H) \cap(a, b)=\phi
$$

if and only if

$$
L(E)>0 \text { for almost all energies } E \in(a, b)
$$

(again assuming the underlying transformation $T$ ergodic).

For both (1.1) and 2.16), the Lyapunov exponent satisfies

$$
L(E) \geq \max (0, \log |\lambda|) .
$$

Moreover, for the almost Mathieu operator, (2.14) becomes an equality if

$E \in \operatorname{Spec} H$, which in view of Katani's theorem is consistent with (2.13)-(2.15). Note however that while positivity of the Lyapunov exponent excludes ac spectrum, it does not necessarily imply point spectrum. For random potentials, the Lyapunov exponents are positive, also at small disorder. The same is conjectured to be true for the skew-shift potential (2.16), which is a central issue in this discussion. Establishing positivity of the Lyapunov exponents of (2.16) (at a given $\lambda$ ) immediately implies absence of ac spectrum. However, it is also the first step in a multi-scale analysis leading to Anderson localization, so far only proven for large $\lambda$. Going one step further, sufficient information about the function

$$
\frac{1}{n} \log \left\|M_{n}(E ; x, y)\right\|
$$

of the three variables $(E, x, y)$ at a sufficiently large scale $n$ would already suffice for this purpose. In a similar vein, it would permit an extension of the theory developed in [K1] to other values of $\lambda$, proving that $\operatorname{Spec} H_{\lambda, \omega, x, y}$ is not a Cantor set. While in principle a computer assisted approach to some of the conjectures above may be possible because it only involves the analysis at some fixed scale, the technical difficulties are considerable. First, one would have to determine an appropriate formulation of the bootstrap process and the ncessary numerical input at some fixed cale $n$. This bootstrap argument depends on a rather lengthy and sophisticated mathematical analysis that would have to be improved. Also, the required scale $n$ may be computationally infeasible. On a much more modest level, it turns out that the numerical evaluation of $L_{n}(E)$ for large $n$ is already a nontrivial 
task, due to the accumulation of errors in calculating the matrix products. Such numerics were carried out several years ago by W. Schlag (private communication) and turned out to be inconclusive at large scale for the above reason.

A few more comments on 'pseudo-random' potentials: while the skew shift $T$ discussed above is an example of a weakly mixing potential, deterministic strongly mixing potentials may be obtained, considering for instance the doubling map $x \mapsto$ $2 x$ acting on $\mathbb{T}$ or a hyperbolic toral automorphism $A \in S L_{2}(\mathbb{Z})$ acting on $\mathbb{T}^{2}$.

Thus one would define

$$
V_{n}=2 \lambda \cos \pi 2^{n} \omega
$$

in the first case and

$$
V_{n}=2 \lambda \cos \left\langle A^{n}\left(\begin{array}{l}
x \\
y
\end{array}\right), e_{1}\right\rangle
$$

in the second setting. For these models, a closer analogy with the random Schrödinger operator theory may be proven. In particular, at small $\lambda$, one obtains positivity of the Lyapunov exponents and Anderson localization. However, the underlying techniques do not seem applicable to skew shift dynamics.

For the operator (1.3), (1.6) with $\beta>1$ and $\beta \notin \mathbb{Z}$ (this last assumption is essential), the semi-continuity methods from $[\mathrm{L}-\mathrm{S}$ may be applied. In addition to (1.7), it was proven in [K3] that for $H_{f, \beta}$ defined in (1.6] and $1 \leq r<\beta<$ $r+1, r \in \mathbb{Z}$,

$$
\sum_{a c}\left(H_{f, \beta}\right) \subset \bigcap_{a_{0}, a_{1}, \ldots, a_{r} \in \mathbb{R}} \sum_{a c}\left(H_{a_{0}, a_{1}, \ldots, a_{r}}\right)
$$

where $H_{a_{0}, \ldots, a_{r}}$ is the Schrödinger operator with potential $f\left(\sum_{j=0}^{r} a_{j} n^{j}\right)$.

The results from [K4 have further implications on the Lyapunov exponents of 1.6. In particular, for (1.3), i.e. $f(t)=2 \lambda \cos t$, Lyapunov exponents do not vanish for all energies $E \notin \mathcal{E}_{\lambda}$, where $\mathcal{E}_{\lambda} \subset \mathbb{R}$ is a set satisfying $\left|\mathcal{E}_{\lambda}\right| \rightarrow 0$ for $\lambda \rightarrow 0$.

\section{Finite SCALE RESTRictions}

It is possible to derive spectral information for te full Hamiltonian $H$ by studying its restriction to finite boxes.

Given an interval $I$ in $\mathbb{Z}$, denote $H_{I}$ the finite matrix (indexed by $I$ ) defined by

$$
H_{I}(m, n)=H(m, n) \text { for } m, n \in I \text {. }
$$


Thus, if for instance $I=[0, N-1]$ and $H$ is given by 2.1],

$$
H_{I}=\left[\begin{array}{cccccc}
V_{0} & 1 & 0 & 0 & \ldots & 0 \\
1 & V_{1} & 1 & 0 & \ldots & 0 \\
0 & 1 & V_{2} & 1 & \ldots & 0 \\
0 & 0 & 1 & V_{3} & & \vdots \\
\vdots & \vdots & 0 & 1 & \ddots & 1 \\
\vdots & \vdots & \vdots & \vdots & & \\
0 & 0 & 0 & 0 & & 1 V_{n-1}
\end{array}\right]
$$

We will need a few elementary results that relate $\operatorname{Spec} H$ with Spec $H_{I}$ and can be used for numerical calculations. They are well-known and we record them here in the explicit form needed.

Lemma 1. Let $I=[a, a+N-1] \subset \mathbb{Z}$.

$$
\begin{gathered}
\text { Let } E \in \mathbb{R}, \xi=\sum_{n \in I} \xi_{n} e_{n},\|\xi\|=\left(\sum_{n \in I}\left|\xi_{n}\right|^{2}\right)^{\frac{1}{2}}=1 \text { such that } \\
\left\|H_{I} \xi-E \xi\right\|<\varepsilon .
\end{gathered}
$$

Then

$$
\operatorname{dist}(E, \operatorname{Spec} H)<\varepsilon+\left|\xi_{a}\right|+\left|\xi_{a+N-1}\right| .
$$

Proof. Define the vector $\eta \in \ell^{2}(\mathbb{Z})$ by

$$
\left\{\begin{array}{l}
\eta_{n}=\xi_{n} \text { if } n \in I \\
\eta_{n}=0 \text { if } n \in \mathbb{Z} \backslash I .
\end{array}\right.
$$

Obviously $\|\eta\|=\|\xi\|=1$.

We compute $H_{\eta}$

$$
(H \eta)_{n}=V_{n} \eta_{n}+\eta_{n+1}+\eta_{n-1}=\left\{\begin{array}{l}
V_{n} \xi_{n}+\xi_{n+1}+\xi_{n-1}=\left(H_{I} \xi\right)_{n} \text { if } a<n<a+N-1 \\
V_{a} \xi_{a}+\xi_{a+1}=\left(H_{I} \xi\right)_{a} \text { if } n=a \\
V_{a+N-1} \xi_{a+N-1}+\xi_{a+N-2}=\left(H_{I} \xi\right)_{a+N-1} \text { if } n=a+N-1 \\
\xi_{a} \text { if } n=a-1 \\
\xi_{a+N-1} \text { if } n=a+N \\
0 \text { if } n<a-1 \text { or } n>a+N .
\end{array}\right.
$$

Hence

$$
H \eta=\sum_{n \in I}\left(H_{I} \xi\right)_{n} e_{n}+\xi_{a} e_{a-1}+\xi_{a+N-1} e_{a+N}
$$

and

$$
\begin{aligned}
H \eta-E \eta & =\sum_{n \in I}\left(\left(H_{I} \xi\right)_{n}-E \xi_{n}\right) e_{n}+\xi_{a} e_{a-1}+\xi_{a+N-1} e_{a+N} \\
\|H \eta-E \eta\| & \leq\left\|H_{I} \xi-E \xi\right\|+\left|\xi_{a}\right|+\left|\xi_{a+N-1}\right|
\end{aligned}
$$




$$
<\varepsilon+\left|\xi_{a}\right|+\left|\xi_{a+N-1}\right|
$$

Conclusion (3.2) then follows from (3.4) and the spectral theorem.

As an immediate consequence, we get

\section{Corollary 2.}

Let $I=[a, a+N-1] \subset \mathbb{Z}$.

Let $\xi^{(1)}, \ldots, \xi^{(N)}$ be the normalized eigenvectors of $H_{I}$ and $\lambda_{1}, \ldots, \lambda_{N}$ the corresponding eigenvalues.

Then, for each $j=1, \ldots, N$

$$
\operatorname{dist}\left(\lambda_{j}, \operatorname{Spec} H\right) \leq\left|\xi_{a}^{(j)}\right|+\left|\xi_{a+N-1}^{(j)}\right|
$$

This property is clearly of interest in order to establish an upperbound on possible gaps in the spectrum of $H$, by considering eigenvalues and eigenvectors of a restriction $H_{I}$. In view of (3.5), only those eigenvalues of $H_{I}$ are of interest for which the corresponding eigenvector is small at the edges of the box $I$. Recall also that for the skew-shift Schrödinger operator, one expects strong localization of the eigenvectors so the boundary contribution for most of them should be quite small.

Next, we prove in some sense a converse property.

\section{Lemma 3.}

Let $N \geq 2$ be a positive integer. Let $0<\varepsilon<1$ be arbitrary.

If $E \in \operatorname{Spec} H$, then there is an interval $I \subset \mathbb{Z}$ of size $N$ and an eigenvalue $E^{\prime}$ of $H_{I}$ such that

$$
\left|E-E^{\prime}\right|<\sqrt{\frac{2}{N}}+\varepsilon
$$

Proof. Since $E \in \operatorname{Spec} H$, there is a finitely supported vector $\eta=\sum^{\prime} \eta_{n} e_{n},\|\eta\|=1$ such that

$$
\|H \eta-E \eta\|<\frac{\varepsilon}{4} .
$$

Let $I=[a, a+N-1]$, with $a \in \mathbb{Z}$ to be specified, and define the vector $\eta^{\prime}=\sum_{n \in I} \eta_{n} e_{n}$. Then

$$
\left(H_{I} \eta^{\prime}\right)_{n}=\left\{\begin{array}{l}
V_{n} \eta_{n}+n_{n-1}+\eta_{n+1}=(H \eta)_{n} \text { if } a<n<a+N-1 \\
V_{a} \eta_{a}+\eta_{a+1}=(H \eta)_{a}-\eta_{a-1} \text { if } n=a \\
V_{a+N-1} \eta_{a+N-1}+\eta_{a+N-2}=(H \eta)_{a+N-1}-\eta_{a+N} \text { if } n=a+N-1 .
\end{array}\right.
$$

Hence

$$
H_{I} \eta^{\prime}-E \eta^{\prime}=\sum_{n=a+1}^{a+N-2}(H \eta-E \eta)_{n} e_{n}+\left(((H-E) \eta)_{a}-\eta_{a-1}\right) e_{a}+\left(((H-E) \eta)_{a+N-1}-\eta_{a+N}\right) e_{a+N-1} .
$$


Therefore

$$
\left\|H_{I} \eta^{\prime}-E \eta^{\prime}\right\|_{2}^{2}=\ell_{1}+\ell_{2}+\ell_{3}
$$

where

$$
\begin{aligned}
& \ell_{1}=\sum_{n=a+1}^{a+N-2}\left|((H-E) \eta)_{n}\right|^{2} \\
& \ell_{2}=\left|((H-E) \eta)_{a}-\eta_{a-1}\right|^{2} \\
& \ell_{3}=\left|((H-E) \eta)_{a+N-1}-\eta_{a+N}\right|^{2} .
\end{aligned}
$$

We claim that we can choose $a \in \mathbb{Z}$ such that

$$
\ell_{1}+\ell_{2}+\ell_{3}<\left(\frac{2+\varepsilon}{N}+\frac{\varepsilon^{2}}{16}\right)\left[\sum_{n=a}^{a+N-1}\left|\eta_{n}\right|^{2}\right]=\left(\frac{2+\varepsilon}{N}+\frac{\varepsilon^{2}}{16}\right)\left\|\eta^{\prime}\right\|_{2}^{2} .
$$

To prove that there exists $a \in \mathbb{Z}$ such that (3.11) holds, simply sum both sides of 3.11 over $a \in \mathbb{Z}$ and show that the left hand side is smaller than the right hand side.

Thus

$$
\begin{aligned}
\sum_{a \in \mathbb{Z}} \ell_{1}=\sum_{a \in \mathbb{Z}} \sum_{n=1}^{N-2}\left|((H-E) \eta)_{a+n}\right|^{2} & = \\
(N-2) \sum_{n \in \mathbb{Z}}\left|((H-E) \eta)_{n}\right|^{2} & =(N-2)\|H \eta-E \eta\|^{2} \\
& <(N-2) \frac{\varepsilon^{2}}{16}
\end{aligned}
$$

by 3.7 .

Next

$$
\begin{aligned}
\sum_{a \in \mathbb{Z}} \ell_{2} & =\sum_{a \in \mathbb{Z}}\left|((H-E) \eta)_{a}\right|^{2} \\
& +\sum_{a \in \mathbb{Z}}\left|\eta_{a-1}\right|^{2} \\
& -2 \operatorname{Re}\left[\sum_{a \in \mathbb{Z}}((H-E) \eta)_{a} \bar{\eta}_{a-1}\right] \\
& \leq\|(H-E) \eta\|^{2}+\|\eta\|^{2}+2 \sum_{a \in \mathbb{Z}}\left|((H-E) \eta)_{a}\right|\left|\eta_{a-1}\right| \\
& <\frac{\varepsilon^{2}}{16}+1+2 \sum_{a \in \mathbb{Z}}\left|((H-E) \eta)_{a}\right|\left|\eta_{a-1}\right| .
\end{aligned}
$$

By the Cauchy-Schwarz inequality

$$
\begin{aligned}
\sum_{a \in \mathbb{Z}}\left|((H-E) \eta)_{a}\right|\left|\eta_{a-1}\right| & \leq\left(\sum_{a \in \mathbb{Z}}\left|((H-E) \eta)_{a}\right|^{2}\right)^{\frac{1}{2}}\left(\sum_{a \in \mathbb{Z}}\left|\eta_{a-1}\right|^{2}\right)^{\frac{1}{2}} \\
& =\|(H-E) \eta\| \cdot\|\eta\|_{2}<\frac{\varepsilon}{4} .
\end{aligned}
$$


Thus

$$
\sum_{a \in \mathbb{Z}} \ell_{2}<1+\frac{\varepsilon^{2}}{16}+2 \cdot \frac{\varepsilon}{4}=\left(1+\frac{\varepsilon}{4}\right)^{2}
$$

and similarly

$$
\sum_{a \in \mathbb{Z}} \ell_{3}<\left(1+\frac{\varepsilon}{4}\right)^{2}
$$

Therefore

$$
\begin{aligned}
\sum_{a \in \mathbb{Z}}\left[\ell_{1}+\ell_{2}+\ell_{3}\right] & <(N-2) \frac{\varepsilon^{2}}{16}+2\left(1+\frac{\varepsilon}{4}\right)^{2} \\
& =N \frac{\varepsilon^{2}}{16}+2\left(1+\frac{\varepsilon}{2}\right) .
\end{aligned}
$$

Summing the right hand side of 3.11 over $a \in \mathbb{Z}$, we obtain indeed

$$
\begin{aligned}
& \left(\frac{2+\varepsilon}{N}+\frac{\varepsilon^{2}}{16}\right) \sum_{a \in \mathbb{Z}}\left(\sum_{n=a}^{a+N-1}\left|\eta_{n}\right|^{2}\right)= \\
& \left(\frac{2+\varepsilon}{N}+\frac{\varepsilon^{2}}{16}\right) N \sum_{n \in \mathbb{Z}}\left|\eta_{n}\right|^{2}=2+\varepsilon+\frac{\varepsilon^{2}}{16} N=3.12 .
\end{aligned}
$$

This proves that we can find some $a \in \mathbb{Z}$ for which 3.11 holds, thus such that

$$
\left\|H_{I} \eta^{\prime}-E \eta^{\prime}\right\|^{2}<\left(\frac{2+\varepsilon}{N}+\frac{\varepsilon^{2}}{16}\right)\left\|\eta^{\prime}\right\|_{2}^{2} .
$$

Define

$$
\xi=\frac{\eta^{\prime}}{\left\|\eta^{\prime}\right\|_{2}}
$$

which satisfies by 3.13

$$
\left\|H_{I} \xi-E \xi\right\|<\left(\frac{2+\varepsilon}{N}+\frac{\varepsilon^{2}}{16}\right)^{\frac{1}{2}}<\sqrt{\frac{2}{N}}+\varepsilon .
$$

This means that there is an eigenvalue $E^{\prime}$ of $H_{I}$ satisfying

$$
\left|E-E^{\prime}\right|<\sqrt{\frac{2}{N}}+\varepsilon
$$

which Proves Lemma 3.

The interest of Lemma 3 is to establish conversely the presence of gaps in Spec $H$. The numerics in this paper also require evaluation of $\sup (\operatorname{Spec} H)$ and $\inf (\operatorname{Spec} H)$ for which we have a slightly better estimate using spectra of finite restrictions.

Lemma 4. Under the assumptions of Lemma 3, there is an interval $I \subset \mathbb{Z}$ of size $N$ and an eigenvalue $E^{\prime}$ of $H_{I}$ such that

$$
E^{\prime}>\sup (\operatorname{Spec} H)-\frac{2}{N}-\varepsilon \text {. }
$$


Proof. We proceed again by an averaging argument.

Denote $I_{a}=[a, a+N-1]$. Clearly

$$
\frac{1}{N} \sum_{a \in \mathbb{Z}} 1_{I_{a}}(x) 1_{I_{a}}(y)=\left\{\begin{array}{l}
1-\frac{|x-y|}{N} \text { if } x, y \in \mathbb{Z},|x-y| \leq N \\
0 \text { if } x, y \in \mathbb{Z},|x-y|>N .
\end{array}\right.
$$

Denote for $\eta \in \ell^{2}(\mathbb{Z})$ by $\eta_{I}$ the vector $\sum_{n \in I} \eta_{n} e_{n}$.

It follows from 3.15 that

$$
\frac{1}{N} \sum_{a \in \mathbb{Z}} H_{I_{a}}=\frac{1}{N} \sum_{a \in \mathbb{Z}} 1_{I_{a}} H 1_{I_{a}}=V+\left(1-\frac{1}{N}\right) \Delta=H-\frac{1}{N} \Delta .
$$

Take $\eta \in \ell^{2}(\mathbb{Z}),\|\eta\|_{2}=1$ such that $\langle H \eta, \eta\rangle>\sup (\operatorname{Spec} H)-\varepsilon$.

It follows that

$$
\frac{1}{N} \sum_{a \in \mathbb{Z}}\left\langle H_{I_{a}} \eta_{I_{a}}, \eta_{I_{a}}\right\rangle=\langle H \eta, \eta\rangle-\frac{2}{N} \operatorname{Re}\left(\sum \eta_{n} \bar{\eta}_{n+1}\right)>\sup (\operatorname{Spec} H)-\varepsilon-\frac{2}{N} .
$$

Also

$$
\frac{1}{N} \sum_{a \in \mathbb{Z}}\left\|\eta_{I_{a}}\right\|_{2}^{2}=1
$$

Hence, there is $a \in \mathbb{Z}$ such that

$$
\left\langle H_{I_{a}} \eta_{I_{a}}, \eta_{I_{a}}\right\rangle>\left(\sup (\operatorname{Spec} H)-\varepsilon-\frac{2}{N}\right)\left\|\eta_{I_{a}}\right\|_{2}^{2}
$$

and the claim in Lemma 4 follows.

Corollary 5. Denote

$$
\sigma_{+}=\sup (\operatorname{Spec} H) \text { and } \sigma_{-}=\inf (\operatorname{Spec} H) .
$$

Then

$$
\sigma_{+} \leq \sup _{|I|=N} \sup \left(\operatorname{Spec} H_{I}\right)+\frac{2}{N} \text { and } \sigma_{-} \geq \inf _{|I|=N} \inf \left(\operatorname{Spec} H_{I}\right)-\frac{2}{N} .
$$

At first sight, the expressions on the r.h.s. of (3.14, (3.16) seem useless for numerics because they involve all intervals $I \subset \mathbb{Z}$ of size $N$.

In the situation of an ergodic Jacobi operator

$$
H^{(x)}=V+\Delta \text { with } V_{n}=f\left(T^{n} x\right)
$$

observe that if

$$
H_{I}^{(x)}=\left(H_{m, n}^{(x)}\right)_{m, n \in I}
$$

then for $I=[a, a+N-1]$, clearly

$$
H_{I}^{(x)}=H_{[0, N-1]}^{\left(T^{a} x\right)} .
$$

Therefore, denoting $\lambda_{+}(x)$, resp $\lambda_{-}(x)$, the largest and smallest eigenvalues of $H_{[0, N-1]}^{(x)}$, we have

$$
\sup _{|I|=N} \sup \left(\operatorname{Spec} H_{I}\right)=\sup _{x} \lambda_{+}(x)
$$


and

$$
\inf _{|I|=N} \inf \left(\operatorname{Spec} H_{I}\right)=\inf _{x} \lambda_{-}(x) .
$$

Consequently, we obtain

Corollary 6. Let $H^{(x)}$ be an ergodic Jacobi operator (3.17) and $\sigma_{+}, \sigma_{-}$defined as in Corollary 5. Let $N \geq 2$ be an integer and $\lambda_{+}(x)$, resp $\lambda_{-}(x)$ the largest and smallest eigenvalue of the $N \times N$-matrix $H_{[0, N-1]}^{(x)}$. Then

$$
\sigma_{+} \leq \max _{x} \lambda_{+}(x)+\frac{2}{N}
$$

and

$$
\sigma_{-} \geq \min _{x} \lambda_{-}(x)-\frac{2}{N} .
$$

In the particular case of the skew shift Schrödinger operator

$$
H=2 \cos 2 \pi n^{2} \omega+\Delta
$$

the underlying dynamics is the skew shift $T$ on $\mathbb{T}^{2}$ mapping $(x, y)$ to $(x+y, y+2 \omega)$. Thus we define

$$
H^{(x, y)}=2 \sum_{n \in \mathbb{Z}} \cos 2 \pi\left(n^{2} \omega+n y+x\right) e_{n} \otimes e_{n}+\Delta
$$

and

$$
H_{N}^{(x, y)}=H_{[0, N-1]}^{(x, y)}=2 \sum_{n=0}^{N-1} \cos 2 \pi\left(n^{2} \omega+n y+x\right) e_{n} \otimes e_{n}+\sum_{n=0}^{N-1}\left(e_{n} \otimes e_{n+1}+e_{n+1} \otimes e_{n}\right) .
$$

Note the following property. Assume $\xi=\sum_{n=0}^{N-1} \xi_{n} e_{n}$ satisfies

$$
H_{N}^{(x, y)} \xi=E \xi
$$

and let $\xi^{\prime}=\sum_{n=0}^{N-1}(-1)^{n} \xi_{n} e_{n}$. Then

$$
H_{N}^{\left(x+\frac{1}{2}, y\right)} \xi^{\prime}=-E \xi^{\prime}
$$

Denoting

$$
\begin{aligned}
& \lambda_{+}=\max _{0 \leq x, y \leq 1} \lambda_{+}(x, y) \\
& \lambda_{-}=\min _{0 \leq x, y \leq 1} \lambda_{-}(x, y)
\end{aligned}
$$

with $\lambda_{+}(x, y)$ and $\lambda_{-}(x, y)$ the largest and smallest eigenvalue of $H_{N}^{(x, y)}$, it follows from the preceding that $\lambda_{-}=-\lambda_{+}$. Hence

Corollary 7. Let $H$ be as in (3.23), $N \geq 2$ and $\lambda_{+}$defined by (3.26). Then

$$
\text { Spec } H \subset\left[-\lambda_{+}-\frac{2}{N}, \lambda_{+}+\frac{2}{N}\right]
$$




\section{Bounding SPECTRAL GAPS FOR THE SKEW-SHIFT POTENTIAL}

Recall the skew-shift Schrödinger operators 1.4

$$
[H u](n)=2\left(\cos 2 \pi n^{2} \omega\right) u_{n}+u_{n+1}+u_{n-1} \text { with } \omega=\frac{1+\sqrt{5}}{2}
$$

and denote

$$
\sigma_{+}=\sup (\operatorname{Spec} H) \quad \sigma_{-}=\inf (\operatorname{Spec} H)=-\sigma_{+} \cdot
$$

We can make a numerical approximation of $\sigma_{+}$by using Corollary 6 . Thus we choose a large $N$ and denote $\lambda_{+}(x, y)$ the largest eigenvalue of $H_{N}^{(x, y)}$ defined by 3.23

$$
H_{N}^{(x, y)}=2 \sum_{n=0}^{N-1} \cos 2 \pi\left(n^{2} \omega+n y+x\right) e_{n} \otimes e_{n}+\sum_{n=0}^{N-1}\left(e_{n} \otimes e_{n+1}+e_{n+1} \otimes e_{n}\right) .
$$

According to 3.26 , one has

$$
\sigma_{+} \leq \max _{0 \leq x, y \leq 1} \lambda_{+}(x, y)+\frac{2}{N}
$$

Once we established an upper and lower bound for Spec $H$, we can obtain information about the size of possible gaps from Corollary 2 Thus we consider the restricted operator

$$
H_{N}=H_{N}^{(0,0)}=2 \sum_{n=0}^{N-1}\left(\cos 2 \pi n^{2} \omega\right) e_{n} \otimes e_{n}+\sum_{n=0}^{N-1}\left(e_{n} \otimes e_{n+1}+e_{n+1} \otimes e_{n}\right)
$$

and denote $\lambda_{1} \geq \lambda_{2} \geq \cdots \geq \lambda_{N}$ the eigenvalues of $H_{N}, \xi^{(1)}, \ldots, \xi^{(N)}$ the corresponding normalized eigenvectors.

Our choice of $N$ here does not necessarily have to be the same as in (4.4). It follows from 3.5 that for any $t \in \mathbb{R}$

$$
\operatorname{dist}(t, \operatorname{Spec} H) \leq \min _{1 \leq j \leq N}\left\{\left|t-\lambda_{j}\right|+\left|\xi_{0}^{(j)}\right|+\left|\xi_{N-1}^{(j)}\right|\right\}
$$

with $\xi^{(j)}=\sum_{n=0}^{N-1} \xi_{n}^{(j)} e_{n},\left\|\xi^{(j)}\right\|_{2}=1$.

Denote $\Gamma$ the largest gap in $\operatorname{Spec} H$. Thus

$$
\Gamma=2\left[\max _{-\sigma_{+}<t<\sigma_{+}} \operatorname{dist}(t, \operatorname{Spec} H)\right]
$$

and by 4.5

$$
\Gamma \leq 2 \max _{-\sigma_{+}<t<\sigma_{+}} \min _{\leq j \leq N}\left\{\left|t-\lambda_{j}\right|+\left|\xi_{0}^{(j)}\right|+\left|\xi_{N-1}^{(j)}\right|\right\} .
$$

Instead of $H_{N}^{(0,0)}$, we may as well consider $H_{N}^{(x, y)}$. Hence

$$
\Gamma \leq 2 \max _{-\sigma_{+}<t<\sigma_{+}} \min _{x, y} \min _{1 \leq j \leq N}\left\{\left|t-\lambda_{j}\right|+\left|\xi_{0}^{(x, y, j)}\right|+\left|\xi_{N-1}^{(x, y, j)}\right|\right\}
$$




\section{Numerics}

Considering the Harper model (1.5) and the skew-shift model 1.4 with $\omega=$

$\frac{1}{2}(\sqrt{5}-1)$, our purpose is to explore the finite scale behavior of the spectra and eigenvectors and compare them for these two models. Our numerics are based on the package MATLAB.

\subsection{Eigenvalues and eigenvectors for the Harper model}

Set

$$
H_{N}=2 \sum_{n=0}^{N-1}(\cos 2 \pi n \omega) e_{n} \otimes e_{n}+\sum_{n=0}^{N-1}\left(e_{n} \otimes e_{n+1}+e_{n+1} \otimes e_{n}\right)
$$

The display below shows the eigenvalue structure at $N=100,200,300,400$, and 500 .

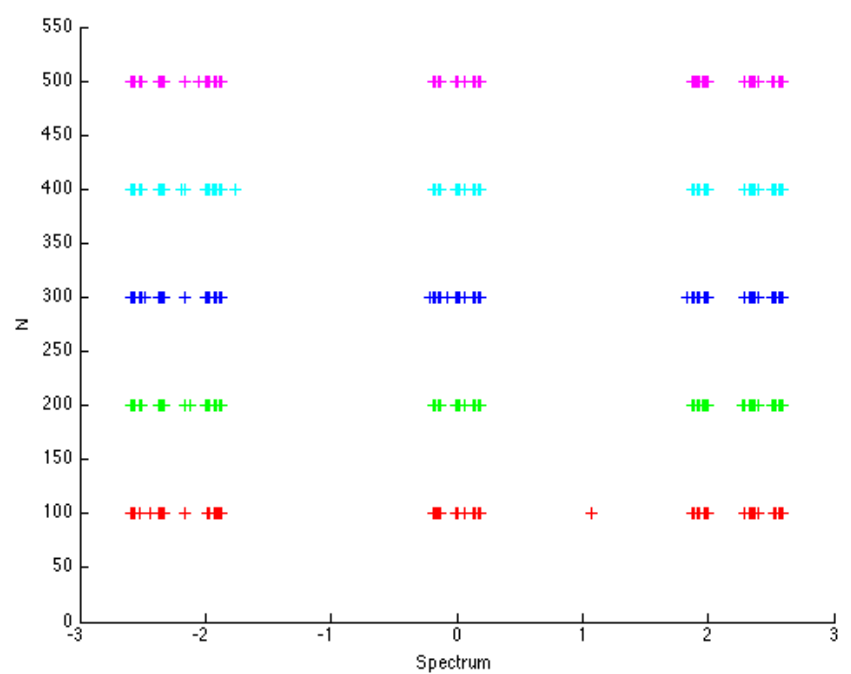

FiguRE 1. Spectrum for the Harper Model

Note the persistency of gaps, in agreement with the (rigorously proven) Cantor structure of the spectrum of (1.1), which for $\lambda=1$ is in fact of zero Lebesque measure.

Next we examine the normalized eigenvectors of $(5.2)$ for $N=200$ in different parts of the spectrum. 


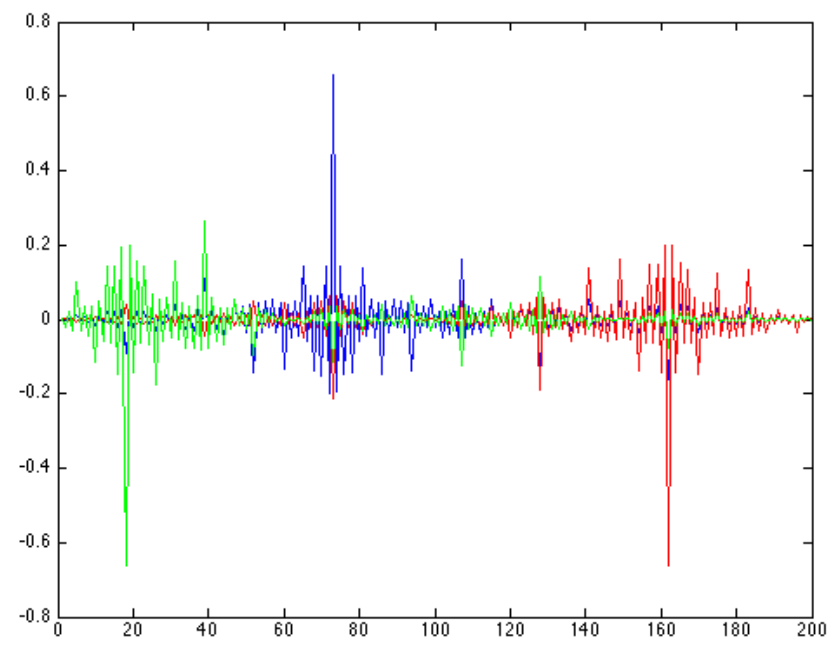

Figure 2. Eigenvectors from the Left Edge of the Spectrum

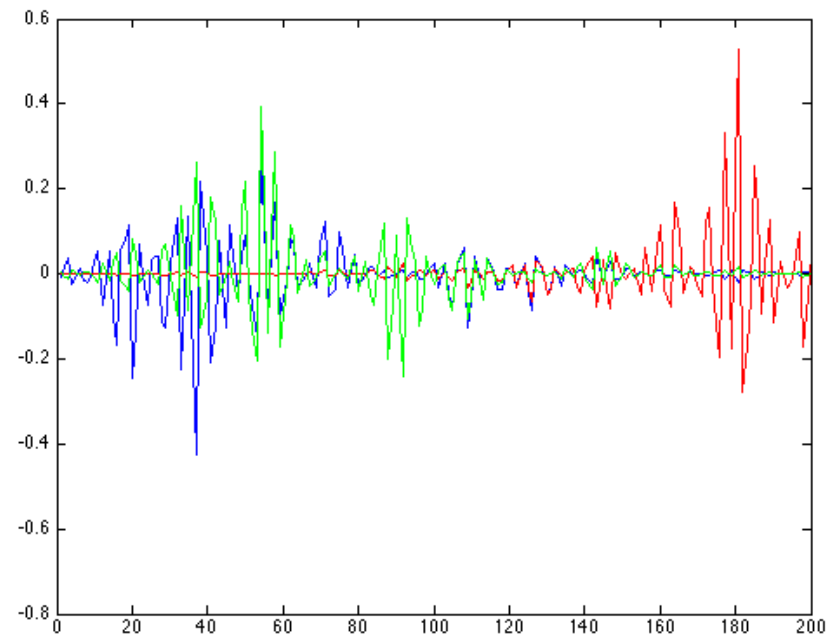

Figure 3. Eigenvectors from the Center of the Spectrum 


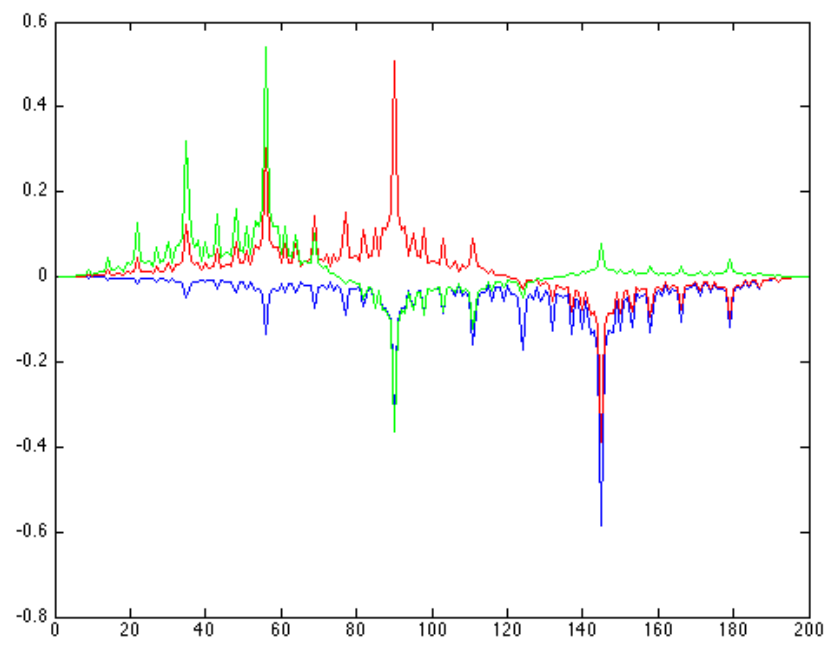

Figure 4. Eigenvectors from the Right Edge of the Spectrum

\subsection{Eigenvalues and eigenvectors for the skew shift model}

Now set

$$
H_{N}=2 \sum_{n=0}^{N-1}\left(\cos 2 \pi n^{2} \omega\right) e_{n} \otimes e_{n}+\sum_{n=0}^{N-1}\left(e_{n} \otimes e_{n+1}+e_{n+1} \otimes e_{n}\right) .
$$

The eigenvalue behavior turns out to be very different as the gaps tend to close for large $N$, in agreement with the conjecture that the spectrum of $(1.4)$ has no gaps. 




Figure 5. Spectrum for Skew Shift Model

Also note that the shape of the eigenvectors is in agreement with the conjecture that (1.4) has localized states. Below some plots for (5.2) at $N=200$ in the center and the edges of the spectrum.

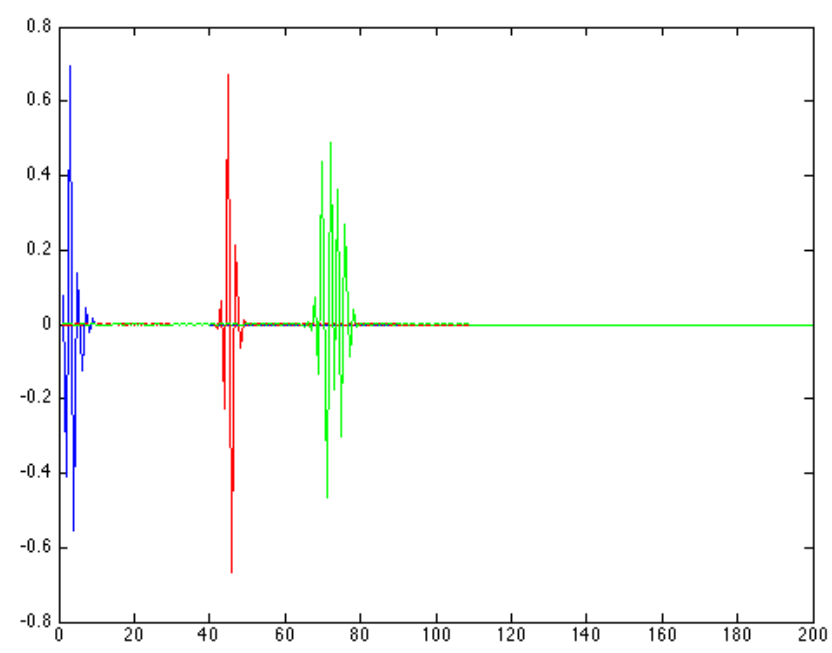

Figure 6. Eigenvectors from Left Edge of Spectrum 


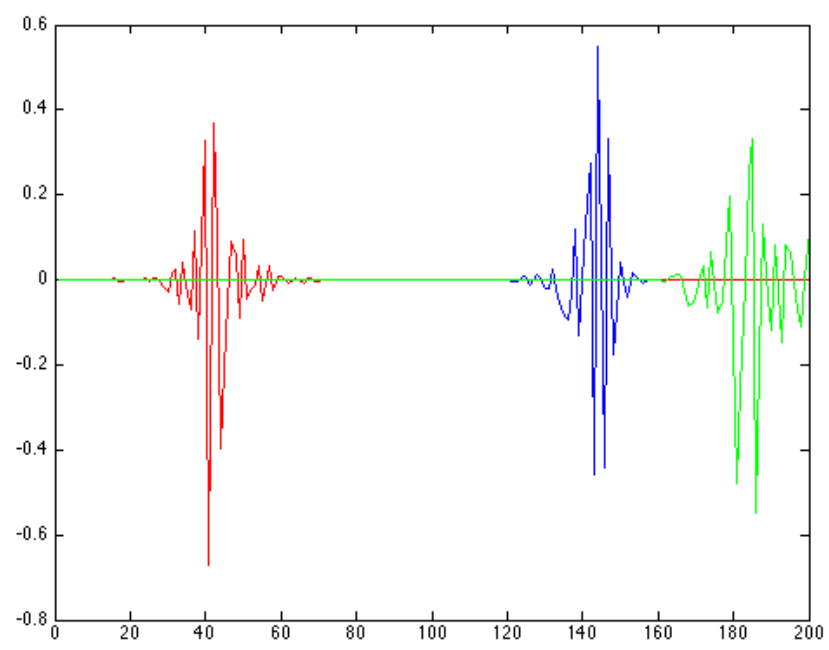

Figure 7. Eigenvectors from Center of Spectrum

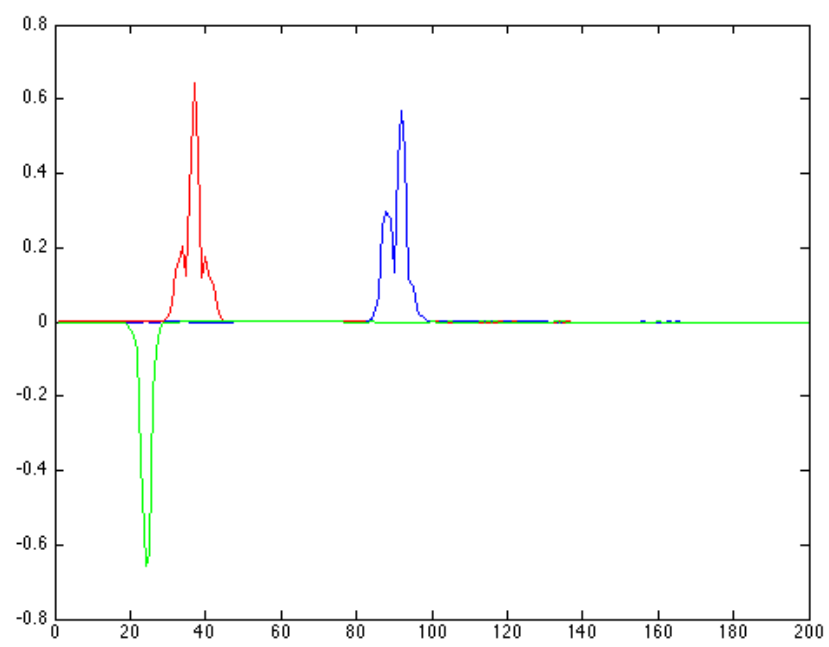

FiguRE 8. Eigenvectors from Right Edge of Spectrum

The localized behavior is already visible at relatively low scale, as is apparent from the collective displays at $N=50, N=100$. 


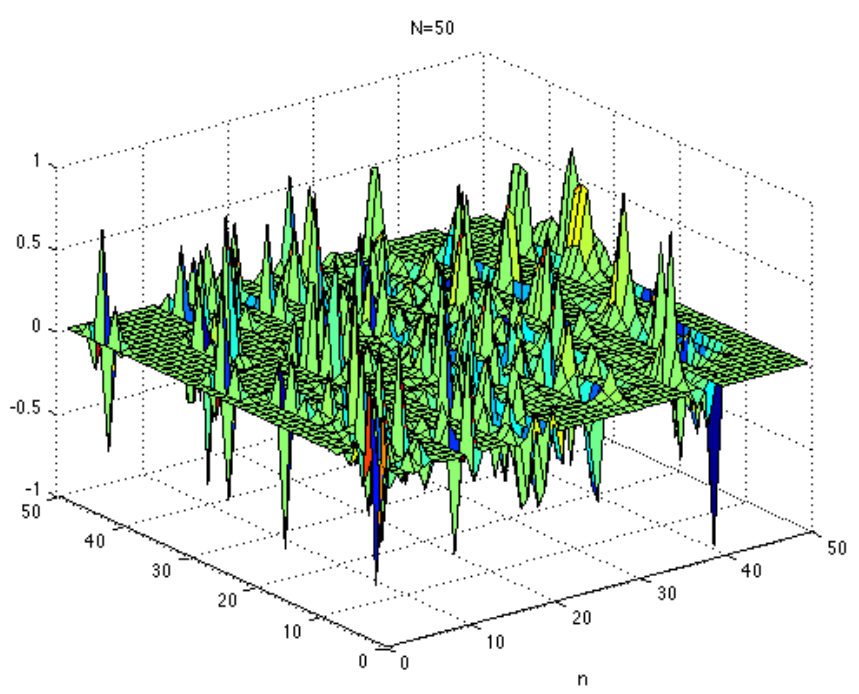

Figure 9. Eigenvectors for Skew Shift Model, N=50

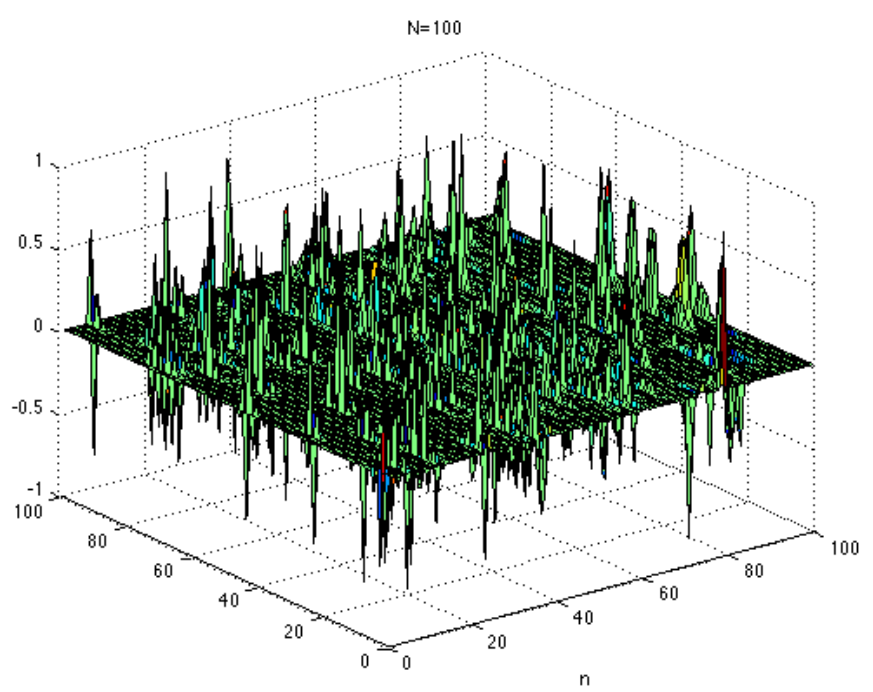

Figure 10. Eigenvectors for Skew Shift Model, N=100

\subsection{Bounding the gaps in the spectrum}

We performed some numerics pertaining to the skew shift model 4.1 as suggested by the discussion in $\S 4$, considering the matrices $H_{N}^{(x, y)}$ as defined by 4.2 . 
The first issue is a numerical evaluation of the maximum $\sigma_{+}$of the spectrum, based on 4.3. We obtained

$$
\sigma_{+} \approx 3.430
$$

The next display graphs the function

$$
\min _{x, y} \min _{1 \leq j \leq N}\left\{\left|t-\lambda_{j}\right|+\left|\xi_{0}^{(x, y, j)}\right|+\left|\xi_{N-1}^{(x, y, j)}\right|\right\}
$$

with $\left\{\lambda_{j}\right\}$ the eigenvalues of $H_{N}^{(x, y)}$ and $\left\{\xi^{(x, y, j)}\right\}$ the corresponding normalized eigenvectors.

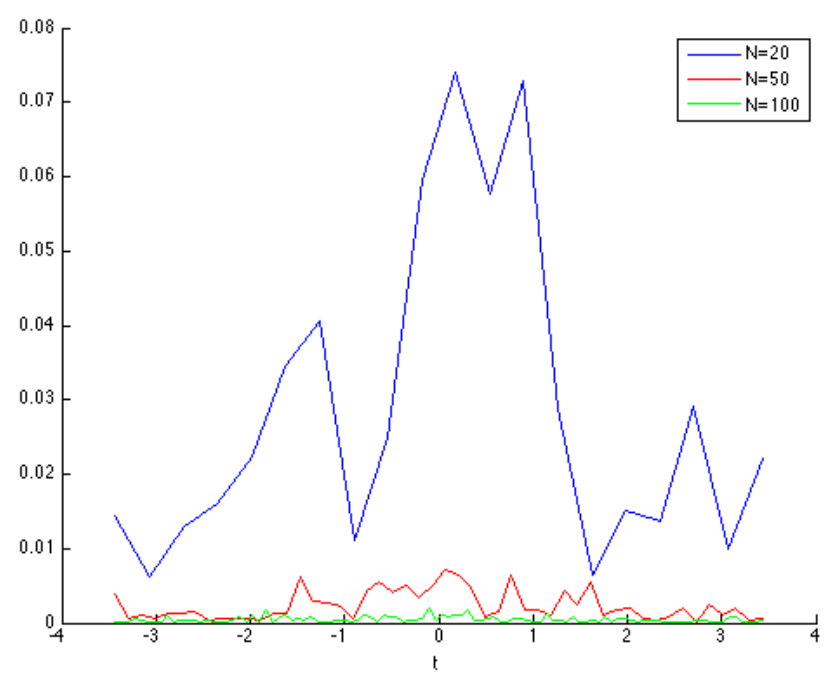

Figure 11. Distance to the Spectrum for Skew Shift Model

Based on 4.8, an upper bound on the largest gap in the spectrum of 4.1

$$
\Gamma<5.708 * 10^{-4}
$$

was obtained. Recall that $\Gamma=0$ according to the conjecture.

As a measure of comparison, we carried out these same numerics for the Harper model (1.4). The maximum of the spectrum now appears to be

$$
\sigma_{+} \approx 2.5975
$$

The function (5.4) for the Harper model is plotted in Figure 4(b).

and a numerical estimate

$$
\Gamma \approx 1.683
$$

for the largest gap is obtained. 


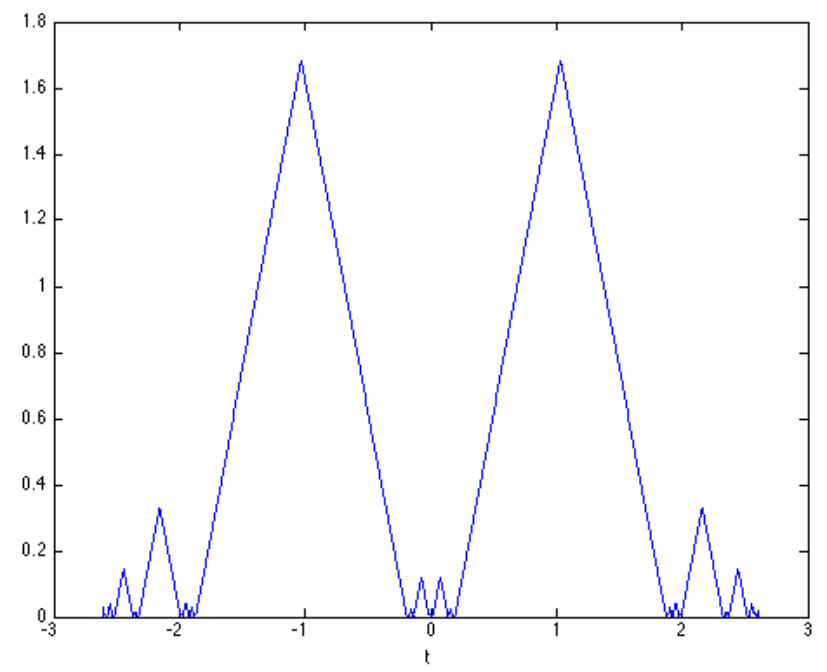

Figure 12. Distance to the Spectrum for Harper Model

\subsection{Lyapunov exponents}

Using the notation from $\S 2$, we computed the function

$$
\frac{\log \left\|M_{N}(E)\right\|}{N}
$$

where $M_{N}(E)$ is the transfer matrix given by 2.18 and potential

$$
\begin{gathered}
V_{j}=2 \cos 2 \pi j^{2} \omega \text { for the skew shift } \\
V_{j}=2 \cos 2 \pi j \omega \text { for the Harper model }
\end{gathered}
$$

As was already clear from earlier calculations performed by W. Schlag [S] around 2002, these numerics are quite unstable when $N$ becomes reasonably large (not surprisingly so).

Figure 13 below for (5.9) at $N=20,50,100$ seems consistent.

There is also consistence with the conjecture that the Lyapunov function $L(E)$ given by 2.21 for the skew shift 1.2 remains positive, even at small disorder $\lambda \neq 0$. Recall that this was only rigorously proven for $|\lambda|>1$.

Next in Figure 5(b), the corresponding display for the Harper model (5.10), which is in accordance with the known fact that $L(E)=0$ for $E$ in the spectrum of 1.1 when $|\lambda| \leq 1$, and the fact that this spectrum is of zero Lebesque measure when $|\lambda|=1$. 


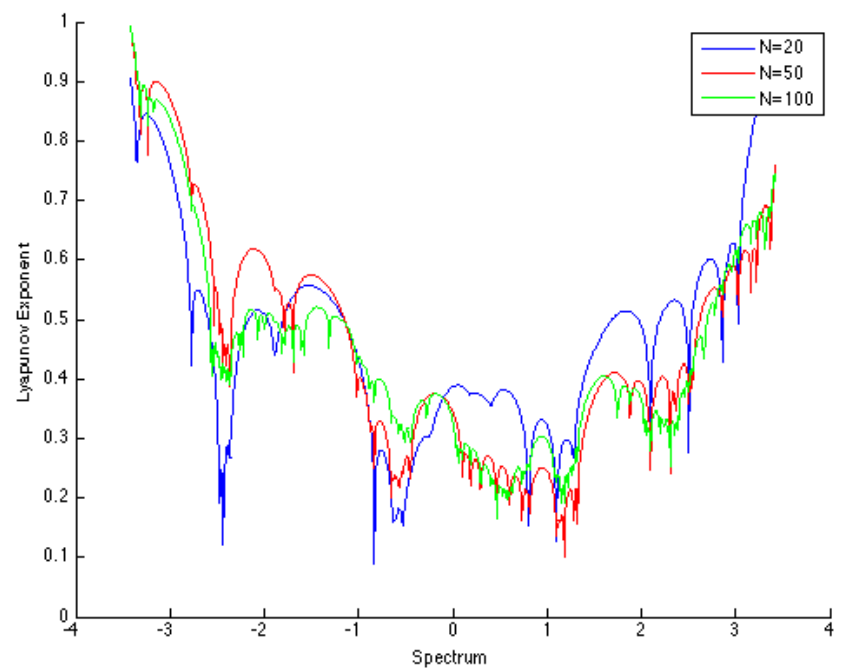

Figure 13. Lyapunov Exponents for Skew Shift Model



FiguRE 14. Lyapunov Exponents for Harper Model

\section{Conclusions}

The numerics carried out for the Schrödinger operator with skew shift potential are in convincing agreement with the general beliefs and conjectures reviewed in the first two sections. In particular, they indicate a spectrum with no gaps, localized eigenstates and positive Lyapunov exponents for all energies. This is in contrast 
with the Harper model where the spectrum is a Cantor set. We have performed these numerics at different scales and obtained consistent results. Our numerics lead moreover to a (rigorous) upperbound on the size of possible gaps (if any) for the skew shift spectrum at the critical coupling, establishing a definitively different spectral structure compared with the Harper model.

In the case of the Harper model, our numerical findings are in accordance with the rigorously proven theoretical results, supporting the reliability of the numerical results. Few such rigorous results are available for the skew-shift counterpart, which makes the present numerical study of interest. Our main finding for the latter model is a strong indication of absence of gaps in the spectrum, as confirmed by an analysis of 'truncated models' at different scales, within computational constraints.

This project offers several further research perspectives. The first is an exploration at larger scales and a finer comparison between random and pseudo-random spectra. Of particular interest is the study of the 'local eigenvalue spacings' in finite models, which are known to be universal and obey Poisson statistics in the random model. One may also wish to consider Schrödinger operators with frequency vectors $\omega$ other than the golden mean (as a test of consistency) and also other couplings $\lambda$. Then one could also numerically analyze Schrödinger operators with different pseudo-random potentials, for instance by replacing in 1.2 the $n^{2}$ by $n^{3}$ etc. (which corresponds to higher order skew shifts) and comparing the results with the present conclusions and discussions from [B-F] and [G-F]. Finally, the problem of positivity of Lyapunov exponents remains an issue to be further studied, technically requiring a balance between scale and computational instability. 


\section{REFERENCES}

[A-J] A. Avila, S. Jitomirskaya, The ten Martini problem, Annals of Math 170 (2009), 303-342).

[B-F] N. Brenner, S. Fishman, Pseudo-randomness and localization, Nonlinearity 4 (1992), 211235.

[F-P] A. Figotin, L. Pastur, Spectra of random and almost periodic operators, Springer-Verlag, 1992.

[G-F] M. Griniasty, S. Fishman, Localization by pseudorandom potentials in one dimension, Phys. Rev, Lett, 60, 1334-1337 (1988).

[G-J-L-S] A. Gordon, S. Jitomirskaya, Y. Last, B. Simon, Duality and singular continuous spectrum in the almost Mathieu equation, Acta Math. 178 (1997), 202, 169-183.

[J] S.Y. Jitomirskaya, Metal-insulator transition for the almost Mathieu operator, Ann. Math. (2) 150(3), 1159-1175 (1999).

[J-K] S. Jitomirskaya, I.V. Krasovsky, Continuity of the measure of the spectrum for discrete quasi-periodic operators, Math. Res. Letters 9 (2002), 413-421.

[K1] H. Krüger, The spectrum of skew-shift Schrödinger operator contains intervals, Journal of Funct. Anal. 262 (2012), 203, 773-810.

[K2] H. Krüger, An explicit example of a skew-shift Schrödinger operator with positive Lyapunov exponent at small coupling, arXiv:1206:1362.

[K3] H. Krüger, A family of Schrödinger Operators whose spectrum is an interval, Comm. Math. Phys. 290:3, 935-939 (2009).

[K4] H. Krüger, Probabilistic averages of Jacobi operators, Comm. Math. Phys. 295:3 (2010).

[L] Y. Last, Almost everything about the almost Mathieu operator. I, In: XIth International Congress of Mathematical Physics, Paris, 1994, pp. 366-372.

[L-S] Y. Last, B. Simon, Eigenfunctions, transfer matrices, and absolutely continuous spectrum of one-dimensional Schrödinger operators, Invent. Math. 135 (1999), 329-367.

[P] R. Peierls, Zur theorie des Diamagnetismus von Leitungselektronen, Z. Phys. 80 (1933), $763-791$

[S] W. Schlag, Private Communication.

Mechanical Engineering Department, University of California, Berkeley, CA 94720

E-mail address: ebc@berkeley.edu 